\title{
Introduction to the special section on Chorus: Chorus and its role in space weather
}

\author{
Bruce T. Tsurutani, ${ }^{1,2}$ Richard B. Horne, ${ }^{3}$ Jolene S. Pickett, ${ }^{4}$ Ondrej Santolik, ${ }^{5,6}$ \\ David Schriver, $^{7}$ and Olga P. Verkhoglyadova ${ }^{1,8}$
}

Received 30 June 2010; accepted 30 June 2010; published 2 September 2010.

[1] Chorus, a naturally occurring magnetospheric electromagnetic wave, is believed to be the result of a long chain of physical processes starting with supergranular circulation at the sun. The interaction of chorus with energetic electrons produces $\sim \mathrm{MeV}$ "killer" electrons that can disable Earth orbiting spacecraft.

Citation: Tsurutani, B. T., R. B. Horne, J. S. Pickett, O. Santolik, D. Schriver, and O. P. Verkhoglyadova (2010), Introduction to the special section on Chorus: Chorus and its role in space weather, J. Geophys. Res., 115, A00F01, doi:10.1029/2010JA015870.

[2] Many of the following papers are a result of the "First International Workshop of Electromagnetic Chorus Plasma Waves" held in La Jolla, California, 10-12 February 2009. The many facets of this esoteric electromagnetic plasma wave were discussed by active researchers in the field at the Workshop. Experimentalists (both ground and satellite observations), computer modelers and theorists discussed their latest findings and had intense interactions with their fellow attendees.

[3] One might ask "why is so much effort being put into such an apparently esoteric subject"? One answer is that it has been speculated by the community, that chorus interacts resonantly with the high-end tail of substorm injected electrons and may produce the relativistic "killer" electrons trapped within the Earth's magnetosphere [Horne et al., 2005; Tsurutani et al., 2006]. These killer electrons appear in the magnetosphere during high speed solar wind streams. We therefore need to know everything we can about these waves to be able to properly model concomitant waveparticle interactions and the process of electron acceleration to these higher energies.

[4] The full chain of physical processes from the Sun to the magnetosphere is quite complex. It is thought that supergranule circulation at the sun produces nonlinear Alfvén waves that are carried to Earth by high speed solar wind streams (that emanate from coronal holes). The southward component of

\footnotetext{
${ }^{1}$ Jet Propulsion Laboratory, California Institute of Technology, Pasadena, California, USA

${ }^{2}$ Institut für Geophysik und Extraterrestrische Physik, Technische Universität Braunschweig, Braunschweig, Germany.

${ }^{3}$ British Antarctic Survey, Natural Environment Research Council, Cambridge, UK

${ }^{4}$ Department of Physics and Astronomy, University of Iowa, Iowa City, Iowa, USA.

${ }^{5}$ Department of Space Physics, Institute of Atmospheric Physics, Prague, Czech Republic.

${ }^{6}$ Faculty of Mathematics and Physics, Charles University, Prague, Czech Republic.

${ }^{7}$ Institute of Geophysics and Planetary Physics, University of California, Los Angeles, California, USA.

${ }^{8}$ CSPAR, University of Alabama in Huntsville, Huntsville, Alabama, USA.

Copyright 2010 by the American Geophysical Union. 0148-0227/10/2010JA015870
}

the Alfvén wave magnetic fields cause magnetic reconnection at the Earth's dayside magnetopause. This leads to energetic plasma injections into the midnight sector of the magnetosphere. The injection of 1-100 keV anistropic electrons leads to a plasma instability, which then generates the chorus.

[5] Who could possibly come up with a hypothesis that long period (hrs) fluid stirrings at the surface of the sun could generate relativistic electrons at a distance $1 \mathrm{AU}$ away? Such a wild hypothesis seems beyond imagination. However, that is what the data analysis and modeling results seem to be implying. Thus, the effort of hundreds of independent scientists studying the various solar, interplanetary, and magnetospheric physics parts of this "space weather" chain, have given us this hypothesis to work with. It is clear that no one person or small group of persons could have derived this complex linkage by themselves. The point that we make is that independent, pure research in many diverse areas is essential toward the understanding of this space weather effect. This special section follows in this scientific tradition. Through more sophisticated instrumentation, theoretical investigations and modeling, the following papers expand our knowledge of chorus and are a testament for it being of fundamental importance in space plasma processes.

[6] Acknowledgment. Portions of the research were performed at the Jet Propulsion Laboratory, California Institute of Technology under contract with NASA.

\section{References}

Horne, R. B., et al. (2005), Wave acceleration of electrons in the Van Allen radiation belts, Nature, 437, 227, doi:10.1038/nature03939.

Tsurutani, B. T., et al. (2006), Corotating solar wind streams and recurrent geomagnetic activity: A review, J. Geophys. Res., 111, A07S01, doi:10.1029/2005JA011273.

R. B. Horne, British Antarctic Survey, Natural Environment Research Council, Madingley Road, Cambridge CB3 0ET, UK.

J. S. Pickett, Department of Physics and Astronomy, University of Iowa, Iowa City, IA 52242, USA.

O. Santolik, Department of Space Physics, Institute of Atmospheric Physics, Boční II 1401, 14131 Prague 4, Czech Republic.

D. Schriver, Institute of Geophysics and Planetary Physics, University of California, 3871 Slichter Hall, Box 951567, Los Angeles, CA 90095, USA.

B. T. Tsurutani and O. P. Verkhoglyadova, Jet Propulsion Laboratory, California Institute of Technology, 4800 Oak Grove Dr., Pasadena, CA 91109, USA. (bruce.tsurutani@jpl.nasa.gov) 